\title{
SINGLE-PARTICLE POTENTIAL IN A RELATIVISTIC HARTREE-FOCK MEAN FIELD APPROXIMATION
}

\author{
M. JAMINON, C. MAHAUX and P. ROCHUS
}

Institut de Physique, Université de Liège, B 4000 Liège 1, Belgium

\section{Received 8 December 1980}

Abstract: A relativistic Hartree-Fock mean field approximation is investigated in a model in which the nucleon field interacts with scalar and vector meson fields. The Hartree-Fock potential felt by individual nucleons enters in a relativistic Dirac single-particle equation. It is shown that in the case of symmetric nuclear matter one can always find a potential which is fully equivalent to the most general mean field and which is only the sum of a Lorentz scalar, of one component of a Lorentz

- tensor and of the fourth component of a Lorentz vector. A non-relativistic potential is derived which yields exactly the same single-particle energies and elastic scattering phase shifts as the relativistic Hartree-Fock potential. Analytical results are presented in the case of nuclear matter. A loca density approximation is constructed which enables one to consider finite nuclei. The input parameters of the model can be chosen in such a way that the empirical saturation properties of nuclear matter are well reproduced. Good agreement is obtained between the calculated nonrelativistic potential and the empirical value of the real part of the optical-model potential at low and at intermediate energy. At intermediate energy, the wine-bottle bottom shape which had previously been found for the potential in the framework of the relativistic Hartree approximation is maintained when the Fock contribution is included.

\section{Introduction}

Relativistic quantum field models of finite nuclei and of nuclear matter are presently receiving considerable attention, see e.g. refs. ${ }^{1-17}$ ). In these models, the nucleon field is coupled to massive meson fields. Most calculations only include two types of mesons, namely a neutral scalar meson $(\sigma)$ and a neutral vector meson $(\omega)$, and are limited to one or another version of a mean field approximation. Despite these drastic simplifications, these models yield fair agreement with quite a number of experimental data provided that the few input parameters (masses and coupling constants) are suitably chosen. These simple models provide a fruitful testing ground for investigating relativistic effects. These effects appear to be quite large, essentially because the familiar shallow non-relativistic shell-model potential results from a cancellation between strong components with well-defined Lorentz tensorial character. This characteristic feature is shared by the available more complicated versions of the model, in which other meson fields are taken into account ${ }^{15,17}$ ) or in 
which ladder summations of Brueckner type are performed ${ }^{15}$ ). It is the existence of these strong components which call for the use of a relativistic approach despite the fact that the nucleon velocity is much smaller than the velocity of light. In particular, these strong components inhibit the derivation of a reliable non-relativistic limit ${ }^{14}$ ). It therefore appears of real interest to investigate in some detail the typically relativistic aspects of the model. These aspects can best be studied in a simple version of the model: here, we investigate the case of nuclear matter and only include the $\sigma$ and $\omega$-mesons.

Ref. ${ }^{14}$ ) deals with the approximation that Miller and Green ${ }^{1}$ ) and Brockmann and Weise ${ }^{8,9}$ ) call the relativistic Hartree model and which turns out (subsect. 2.2) to be identical to Walecka's mean field approximation ${ }^{3}$ ) in the case of nuclear matter. In the present work, we extend this previous study to include Pauli exchange, i.e. we analyze the relativistic Hartree-Fock approximation. We mainly investigate the predictions of the model concerning the familiar local non-relativistic nucleon-nucleus potential, which is well-known empirically from many successful applications "of the shell model and of the optical model. Here, we define this non-relativistic potential by the requirements that it be local and "equivalent" to the relativistic Hartree-Fock potential, equivalent in the sense that it yields the same bound state energies and elastic scattering phase shifts. This had previously been performed in the case of the Hartree approximation ${ }^{9,14}$ ), but we shall see that new features arise from the inclusion of the non-local Fock contribution. The existence of non-local components appears to be required on purely phenomenological grounds ${ }^{18}$ ) and the present investigation of the Fock contribution is thus of real interest. The Fock contribution has been considered by Miller ${ }^{2}$ ) and by Brockmann and Weise ${ }^{17}$ ) in the case of finite nuclei. Here, we mainly discuss nuclear matter where many properties become simpler and thus more transparent. Our work differs from those of $\mathrm{Chin}^{6}$ ) and of Bolsterli ${ }^{4}$ ) by the nature of the self-consistency requirements that we impose on the fields. By analogy with Walecka's terminology, we call our approximation the Hartree-Fock mean field approximation.

The present paper is organized as follows. Sect. 2 is devoted to the definition and to the investigation of the relativistic Hartree-Fock mean field approximation, with emphasis on the self-consistency requirements and on the decomposition of the relativistic single-particle potential into components which have well-defined Lorentz-transformation properties. We show that this decomposition is not unique, but that this ambiguity does not affect the observable quantities. Sect. 3 deals with the derivation of the Schrödinger-equivalent potential in finite nuclei and in nuclear matter, and with the construction of a suitable local density approximation. Sect. 4 contains numerical results concerning the relativistic mass operator, the Schrödinger-equivalent potential and the average binding energy per nucleon; some preliminary results have been presented in ref. ${ }^{19}$ ). Sect. 5 contains a brief summary and draws some conclusions.

\section{Relativistic Hartree-Fock mean field approximation}

2.1. THE MODEL

We adopt here the model studied by Brockmann ${ }^{9}$ ), whose notation we closely follow. In this model, the nucleon-nucleon interaction is mediated by the exchange of only two types of mesons, namely the scalar meson $\sigma$ and the vector meson $\omega$. One furthermore drops all terms with annihilation or creation operators for antinucleons. This leads to the following nuclear hamiltonian ${ }^{9}$ )

$$
\begin{aligned}
H= & \sum_{k, k^{\prime}} \int \mathrm{d}^{3} r_{1} f_{k^{\prime}}^{\dagger}\left(\boldsymbol{r}_{1}\right) \gamma^{0}(-i \boldsymbol{\gamma} \cdot \boldsymbol{\nabla}+m) f_{k}\left(\boldsymbol{r}_{1}\right) b_{k^{\prime}}^{\dagger} b_{k} \\
& +\frac{1}{2} \sum_{k, k^{\prime}, q_{,} q^{\prime}} \int \mathrm{d}^{3} r_{1} \mathrm{~d}^{3} r_{2} f_{k^{\prime}}^{\dagger}\left(\boldsymbol{r}_{1}\right) f_{q^{\prime}}^{\dagger}\left(\boldsymbol{r}_{2}\right) V^{k k^{\prime}}\left(\left|\boldsymbol{r}_{1}-\boldsymbol{r}_{2}\right|\right) \\
& \times f_{q}\left(\boldsymbol{r}_{2}\right) f_{k}\left(\boldsymbol{r}_{1}\right) b_{k^{\prime}}^{\dagger} b_{q^{\prime}}^{\dagger} b_{q} b_{k},
\end{aligned}
$$

where $\left\{f_{k}(\boldsymbol{r})\right\}$ denotes a complete set of Dirac spinors with the corresponding creation (annihilation) operators $b_{k}^{\dagger}\left(b_{k}\right)$; the index $k$ generically refers to the quantum numbers of the single-particle state. The nucleon-nucleon interaction reads $(i=\sigma, \omega)$

$$
V^{k k^{\prime}}\left(\left|\boldsymbol{r}_{1}-\boldsymbol{r}_{2}\right|\right)=\sum_{i} \Gamma_{i}(1,2) V_{i}^{k k^{\prime}}\left(\left|\boldsymbol{r}_{1}-\boldsymbol{r}_{2}\right|\right)
$$

with

$$
\begin{aligned}
\Gamma_{\sigma}(1,2)= & -\gamma_{0}(1) \gamma^{0}(2) \\
\Gamma_{\omega}(1,2)= & \gamma_{0}(1) \gamma^{0}(2) \gamma_{\mu}(1) \gamma^{\mu}(2) \\
V_{i}^{k k^{\prime}}(r)= & \frac{g_{i}^{2}}{4 \pi} \frac{\Lambda_{i}^{2}}{\Lambda_{i}^{2}-m_{i}^{2}}\left\{r^{-1} \exp \left[-\left\{m_{i}^{2}-\left(E_{k}-E_{k^{\prime}}\right)^{2}\right\}^{1 / 2} r\right]\right. \\
& \left.-r^{-1} \exp \left[-\left\{\Lambda_{i}^{2}-\left(E_{k}-E_{k^{\prime}}\right)^{2}\right\}^{1 / 2} r\right]\right\}
\end{aligned}
$$

The input parameters of the model are the coupling constants $g_{i}^{2}$, the meson masses $m_{i}$ and the cut-off energies $\Lambda_{i}$. These energies $\Lambda_{i}$ represent phenomenologically the effect of vertex corrections which actually go beyond the Hartree-Fock approach.

The derivation of the Hartree-Fock approximation for doubly magic nuclei is described in refs. $\left.{ }^{1,9}\right)$. It leads to the following single-particle wave equation

$$
\left(\boldsymbol{\alpha} \cdot \boldsymbol{p}+\gamma^{0} m\right) \phi_{k}\left(\boldsymbol{r}_{1}\right)+\gamma^{0} \int \mathrm{d}^{3} r_{2} U^{\mathrm{HF}}\left(\boldsymbol{r}_{1}, \boldsymbol{r}_{2}\right) \phi_{k}\left(\boldsymbol{r}_{2}\right)=E_{k} \phi_{k}\left(\boldsymbol{r}_{1}\right),
$$

or, more explicitly

$$
\left(\boldsymbol{\alpha} \cdot \boldsymbol{p}+\gamma^{0} m\right) \phi_{k}\left(\boldsymbol{r}_{1}\right)+\gamma^{0} U^{\mathrm{H}}\left(r_{1}\right) \phi_{k}\left(\boldsymbol{r}_{1}\right)+\gamma^{0} \int \mathrm{d}^{3} r_{2} U^{\mathrm{F}}\left(\boldsymbol{r}_{1}, \boldsymbol{r}_{2}\right) \phi_{k}\left(\boldsymbol{r}_{2}\right)=E_{k} \phi_{k}\left(\boldsymbol{r}_{1}\right),
$$


where the local Hartree potential is given by

$$
U^{\mathrm{H}}\left(r_{1}\right)=\gamma^{o} \sum_{j \text { occ }} \int \mathrm{d}^{3} r_{2} \phi_{j}^{\dagger}\left(\boldsymbol{r}_{2}\right) V^{i i}\left(\left|\boldsymbol{r}_{1}-\boldsymbol{r}_{2}\right|\right) \phi_{i}\left(\boldsymbol{r}_{2}\right),
$$

while the non-local Fock component reads

$$
U^{\mathrm{F}}\left(\boldsymbol{r}_{1}, \boldsymbol{r}_{2}\right)=-\gamma^{\mathrm{o}} \sum_{j \mathrm{occ}} \phi_{j}^{\dagger}\left(\boldsymbol{r}_{2}\right) V^{k j}\left(\left|\boldsymbol{r}_{1}-\boldsymbol{r}_{2}\right|\right) \phi_{i}\left(\boldsymbol{r}_{1}\right) .
$$

In eqs. (2.7) and (2.8) the sum over $j$ runs over all occupied orbitals. Note that eqs. (2.6a)-(2.8) are formally identical to those encountered in the non-relativistic Hartree-Fock approximation.

\subsection{HARTREE-FOCK MEAN FIELD APPROXIMATION IN NUCLEAR MATTER}

Most of the present paper is devoted to the case of nuclear matter, in which eqs. (2.6a)-(2.8) considerably simplify. Indeed, all spinors then are normalized plane waves:

$$
\phi_{\boldsymbol{k}}(\boldsymbol{r})=u(\boldsymbol{k}) \exp (\boldsymbol{i k} \cdot \boldsymbol{r}),
$$

where we for simplicity drop explicit reference to the spin and isospin variables. The sum over $j$ in eqs. (2.7) and (2.8) runs over values $j=|j|<k_{\mathrm{F}}$; the Fermi momentum $k_{\mathrm{F}}$ is related to the baryon density by the familiar equation

$$
\rho=\frac{2}{3 \pi^{2}} k_{\mathrm{F}}^{3} .
$$

The Hartree component is given by

$$
U^{\mathrm{H}}\left(r_{1}\right)=\gamma^{0} \sum_{j<k_{F}} \int \mathrm{d}^{3} r_{2} u^{\dagger}(\boldsymbol{j}) V^{i j}\left(\left|\boldsymbol{r}_{1}-\boldsymbol{r}_{2}\right|\right) u(\boldsymbol{j}),
$$

while one has

$$
\begin{aligned}
\int \mathrm{d}^{3} r_{2} U^{\mathrm{F}}\left(\boldsymbol{r}_{1}, \boldsymbol{r}_{2}\right) \phi_{\boldsymbol{k}}\left(\boldsymbol{r}_{2}\right)= & -\gamma^{0} \sum_{i<k_{\mathbf{F}}} \int \mathrm{d}^{3} r_{2} u^{\dagger}(j) V^{k j}\left(\left|\boldsymbol{r}_{1}-\boldsymbol{r}_{2}\right|\right) \\
& \times \mathrm{e}^{i(\boldsymbol{k}-\boldsymbol{j}) \cdot\left(\boldsymbol{r}_{2}-\boldsymbol{r}_{1}\right)} u(j) \phi_{k}\left(\boldsymbol{r}_{1}\right), \\
= & U^{\mathrm{F}}(\boldsymbol{k}) \phi_{\boldsymbol{k}}\left(\boldsymbol{r}_{1}\right) .
\end{aligned}
$$

Eq. (2.13) reflects the fact that in a uniform medium the non-local potential $U^{\mathrm{F}}\left(\boldsymbol{r}_{1}, \boldsymbol{r}_{2}\right)$ only depends on $\boldsymbol{r}_{1}-\boldsymbol{r}_{2}$. We have for simplicity used the notation $U^{\mathrm{F}}(k)$ to denote the Fourier transform of $U^{\mathrm{F}}\left(\left|\boldsymbol{r}_{1}-\boldsymbol{r}_{2}\right|\right)$. The quantity $U^{\mathrm{F}}(k)$ is represented by a $4 \times 4$ matrix in spinor space. Note the intimate relationship between momentum $(k)$ dependence and non-locality.

\subsection{SELF-CONSISTENT REQUIREMENTS}

In order to calculate the Hartree-Fock potential we still need to specify the nature of the spinors $u(j)$. In the spirit of the mean field approximation, it appears most natural to take into account the fact that each nucleon feels a self-consistent average potential, i.e. to adopt for $u(j)$ a spinor which is the normalized solution of the Dirac equation

$$
\left\{\boldsymbol{\alpha} \cdot \boldsymbol{k}+\gamma^{0}\left[m+U^{\mathrm{H}}+U^{\mathrm{F}}(\boldsymbol{k})\right]\right\} u(\boldsymbol{k})=E_{k} u(\boldsymbol{k}) .
$$

Let us first consider the Hartree mean field approximation. Then, a few elementary manipulations lead to

$$
u(\boldsymbol{k})=Q_{\mathrm{H}}^{-1}\left(\begin{array}{c}
\left(k^{2}+m_{\mathrm{H}}^{2}\right)^{1 / 2}+m_{\mathrm{H}} \\
0 \\
k_{3} \\
k_{1}+i k_{2}
\end{array}\right)
$$

in the case of a spin-up nucleon. Here, $Q_{\mathrm{H}}$ is a normalization factor chosen such that $u^{\dagger}(k) u(k)=1$, and the effective mass $m_{\mathrm{H}}$ is given by $m_{\mathrm{H}}=m+U_{\mathrm{s}}^{\mathrm{H}}$, with

$$
\begin{gathered}
U_{\mathrm{s}}^{\mathrm{H}}=-\rho_{\mathrm{s}} g_{\sigma}^{2} / m_{\sigma}^{2}, \\
\rho_{\mathrm{s}}=\frac{4}{(2 \pi)^{3}} \int_{0}^{k_{\mathrm{F}}} \mathrm{d}^{3} k \frac{m_{\mathrm{H}}}{\left(k^{2}+m_{\mathrm{H}}^{2}\right)^{1 / 2}} .
\end{gathered}
$$

The Hartree potential is given by

$$
U^{\mathrm{H}}=I U_{\mathrm{s}}^{\mathrm{H}}+\gamma^{0} U_{0}^{\mathrm{H}}
$$

where $I$ is the $4 \times 4$ unit matrix and where

$$
U_{0}^{\mathrm{H}}=\rho g_{\omega}^{2} / m_{\omega}^{2} .
$$

The following dispersion relation connects $k$ and $E_{k}$

$$
\left(E_{k}-U_{0}^{\mathrm{H}}\right)^{2}=k^{2}+\left(m+U_{\mathrm{s}}^{\mathrm{H}}\right)^{2} .
$$

The kinetic + rest mass energy of nucleon $\boldsymbol{k}$ reads

$$
\mathscr{H}(k)=\left\langle\phi_{\boldsymbol{k}}\left|\boldsymbol{\alpha} \cdot \boldsymbol{k}+\gamma^{0} m\right| \phi_{\boldsymbol{k}}\right\rangle=\left(m m_{\mathrm{H}}+k^{2}\right)\left(m_{\mathrm{H}}^{2}+k^{2}\right)^{-1 / 2},
$$

while its potential energy is given by

$$
\mathcal{U}(k)=\left\langle\phi_{k}\left|I U_{0}^{\mathrm{H}}+\gamma^{0} U_{\mathrm{s}}^{\mathrm{H}}\right| \phi_{k}\right\rangle=U_{0}^{\mathrm{H}}+U_{\mathrm{s}}^{\mathrm{H}} m_{\mathrm{H}}\left(k^{2}+m_{\mathrm{H}}^{2}\right)^{-1 / 2} .
$$

The average energy per nucleon is equal to

$$
m+\frac{B}{A}=3 k_{F}^{-3} \int_{0}^{k_{F}} k^{2} \mathrm{~d} k\left[\mathscr{K}(k)+\frac{1}{2} \mathscr{U}(k)\right] .
$$


If one had calculated the nuclear field by using first-order perturbation theory in the coupling constants $g_{i}^{2}$ while neglecting the exchange (Fock) term, one would have obtained eqs. (2.16)-(2.23) for the direct term with, however, $m_{\mathrm{H}}$ everywhere replaced by the bare nucleon mass $m$ [ref. $\left.{ }^{6}\right)$ ]. This first-order approximation could also be called the "Hartree" approximation. Here, however, we have assumed in eq. (2.15) that each nucleon feels the average potential. The corresponding average energy (2.23) is identical to that obtained by Walecka ${ }^{3}$ ) in his "mean field" approximation. That is why we call the present approximation the Hartree mean field approximation.

We now introduce the Hartree-Fock mean field approximation for the average nucleon-nucleus potential. It can be written as the sum of a Hartree and of a Fock component. The Hartree component $U^{\mathrm{H}}$ still has the form (2.18); a few manipulations based on eqs. (2.2)-(2.5) and (2.12), (2.13) show that the Fock (exchange) component $U^{\mathrm{F}}(\boldsymbol{k})$ has the form

$$
U^{\mathrm{F}}(\boldsymbol{k})=I U_{\mathrm{s}}^{\mathrm{F}}(k)+\gamma^{0} U_{0}^{\mathrm{F}}(k)+\boldsymbol{\gamma} \cdot \frac{\boldsymbol{k}}{k} U_{\mathrm{v}}^{\mathrm{F}}(k),
$$

where $U_{\mathrm{s}}^{\mathrm{F}}(k), U_{0}^{\mathrm{F}}(k)$ and $U_{\mathrm{v}}^{\mathrm{F}}(k)$ are scalar functions of $k$. For instance, $U_{\mathrm{s}}^{\mathrm{F}}(k)$ reads $(i=\sigma, \omega)$

$$
U_{\mathrm{s}}^{\mathrm{F}}(k)=-\gamma^{0} \sum_{i} \frac{g_{i}^{2}}{(2 \pi)^{3}} \frac{\Lambda_{i}^{2}}{\Lambda_{i}^{2}-m_{i}^{2}} \int_{0}^{k_{\mathrm{F}}} \mathrm{d}^{3} j\left[\frac{\sum_{\mathrm{s}} u^{(\mathrm{s}) \dagger}(\boldsymbol{j}) \Gamma_{i} u^{(\mathrm{s})}(\boldsymbol{j})}{(\boldsymbol{k}-\boldsymbol{j})^{2}+m_{i}^{2}-\left(E_{k}-E_{j}\right)^{2}}-\left(m_{i} \leftrightarrow \Lambda_{i}\right)\right],
$$

where we wrote explicitly the sum over the spin degrees of freedom (s). Note that $\omega$-exchange also yields a contribution to $U_{\mathrm{s}}^{\mathrm{F}}(k)$. We still have to specify the spinor $u^{(\mathrm{s})}(\boldsymbol{j})$. In keeping with the spirit of the mean field approximation, we take for $u^{(\mathrm{s})}(j)$ the solution of the Dirac equation

$$
\left\{\boldsymbol{\alpha} \cdot \boldsymbol{k}+\gamma^{0}\left[m+U^{\mathrm{H}}+U^{\mathrm{F}}(\boldsymbol{k})\right]\right\} u^{(\mathrm{s})}(\boldsymbol{k})=E_{k} u^{(\mathrm{s})}(\boldsymbol{k}) .
$$

In the case of a spin-up nucleon, for instance, this yields

$$
u(\boldsymbol{k})=Q_{\mathrm{HF}}^{-1}\left(\begin{array}{c}
{\left[k_{\mathrm{v}}^{2}+m_{\mathrm{HF}}^{2}(k)\right]^{1 / 2}+m_{\mathrm{HF}}(k)} \\
0 \\
k_{\mathrm{v}_{3}} \\
k_{\mathrm{v}_{1}}+i k_{\mathrm{v}_{2}}
\end{array}\right),
$$

where

$$
Q_{\mathrm{HF}}=\left\{2\left(k_{\mathrm{v}}^{2}+m_{\mathrm{HF}}^{2}\right)^{1 / 2}\left[\left(k_{\mathrm{v}}^{2}+m_{\mathrm{HF}}^{2}\right)^{1 / 2}+m_{\mathrm{HF}}\right]\right\}^{1 / 2} .
$$

The "effective mass" $m_{\mathrm{HF}}(k)$ reads

$$
m_{\mathrm{HF}}(k)=m+U_{\mathrm{s}}(k),
$$

with

$$
U_{\mathrm{s}}(k)=U_{\mathrm{s}}^{\mathrm{H}}+U_{\mathrm{s}}^{\mathrm{F}}(k)
$$

the vector $\boldsymbol{k}_{\mathrm{v}}$ is given by

$$
\boldsymbol{k}_{\mathrm{v}}=\boldsymbol{k}+U_{\mathrm{v}}^{\mathrm{F}}(k) \frac{\boldsymbol{k}}{k}
$$

The following dispersion relation connects $E_{k}$ and $k$ :

$$
\left[E_{k}-U_{0}(k)\right]^{2}=k_{\mathrm{v}}^{2}+m_{\mathrm{HF}}^{2}(k),
$$

with

$$
U_{0}(k)=U_{0}^{\mathrm{H}}+U_{0}^{\mathrm{F}}(k) .
$$

The explicit expressions of the potentials are the following. The Hartree potential is still given by eqs. (2.16), (2.18) and (2.19), where the scalar density $\rho_{\mathrm{s}}$ is now given by

$$
\rho_{\mathrm{s}}=\frac{4}{(2 \pi)^{3}} \int_{0}^{k_{\mathrm{F}}} \mathrm{d}^{3} j \frac{m_{\mathrm{HF}}(j)}{\left[j_{\mathrm{v}}^{2}+m_{\mathrm{HF}}^{2}(j)\right]^{1 / 2}} .
$$

The Fock potential is given by eq. (2.24) with

$$
\begin{aligned}
U_{\mathrm{s}}^{\mathrm{F}}(k)= & \left\{\frac{g_{\sigma}^{2}}{16 \pi^{3}} \frac{\Lambda_{\sigma}^{2}}{\Lambda_{\sigma}^{2}-m_{\sigma}^{2}} \int_{0}^{k_{\mathrm{F}}} \mathrm{d}^{3} j m_{\mathrm{HF}}(j)\left[j_{\mathrm{v}}^{2}+m_{\mathrm{HF}}^{2}\right]^{-1 / 2}\right. \\
& \left.\times\left(\left[m_{\sigma}^{2}-(k-j)_{\mu}(k-j)^{\mu}\right]-\left[m_{\sigma} \leftrightarrow \Lambda_{\sigma}\right]\right)\right\}-4\{\sigma \leftrightarrow \omega\} \\
U_{0}^{\mathrm{F}}(k)= & \left\{\frac{g_{\sigma}^{2}}{16 \pi^{3}} \frac{\Lambda_{\sigma}^{2}}{\Lambda_{\sigma}^{2}-m_{\sigma}^{2}} \int_{0}^{k_{\mathrm{F}}} \mathrm{d}^{3} j\left(\left[m_{\sigma}^{2}-(k-j)_{\mu}(k-j)^{\mu}\right]^{-1}-\left[m_{\sigma} \leftrightarrow \Lambda_{\sigma}\right]^{-1}\right)\right\} \\
+ & 2\{\sigma \leftrightarrow \omega\}, \\
U_{\mathrm{v}}^{\mathrm{F}}(k)= & \left\{-\frac{g_{\sigma}^{2}}{16 \pi^{3}} \frac{\Lambda_{\sigma}^{2}}{\Lambda_{\sigma}^{2}-m_{\sigma}^{2}} k^{-1} \int_{0}^{k_{\mathrm{F}}} \mathrm{d}^{3} j\left[j_{\mathrm{v}}^{2}+m_{\mathrm{HF}}^{2}\right]^{-1 / 2} k \cdot j_{\mathrm{v}}\right. \\
& \left.\times\left(\left[m_{\sigma}^{2}-(k-j)_{\mu}(k-j)^{\mu}\right]^{-1}-\left[m_{\sigma} \leftrightarrow \Lambda_{\sigma}\right]^{-1}\right)\right\}+2\{\sigma \leftrightarrow \omega\}
\end{aligned}
$$

Bolsterli ${ }^{4}$ ) and Chin $^{6}$ ) performed a calculation of the average potential by using perturbation theory limited to first-order in the coupling constants $g_{i}^{2}$. They obtained the same results as above (with however $\Lambda_{i} \rightarrow \infty$ since they did not introduce cut-off factors), with the difference that everywhere the effective mass $m_{\mathrm{HF}}$ was replaced by the bare nucleon mass $m$ and that moreover the vector $\boldsymbol{j}_{\mathrm{v}}$ is replaced by $\boldsymbol{j}$. As we described, this difference originates from our use of the self-consistent equation (2.27) for the spinors, while Bolsterli and Chin used free plane waves in keeping with 
a first-order calculation. That is why we refer to the present approximation as the Hartree-Fock mean field approximation. The quantitative importance of the selfconsistency will be illustrated in sect. 4 .

\subsection{LORENTZ COMPONENTS OF THE RELATIVISTIC POTENTIAL}

In the present section, we show that there exist several ways of writing the relativistic field $U^{\mathrm{HF}}$ as a sum of terms which have well-defined Lorentz transformation properties. Eqs. (2.18) and (2.24) yield $U^{\mathrm{HF}}$ in the form

$$
U^{\mathrm{HF}}(\boldsymbol{k})=I U_{\mathrm{s}}^{\mathrm{HF}}(k)+\gamma^{0} U_{0}^{\mathrm{HF}}(k)+\boldsymbol{\gamma} \cdot \frac{\boldsymbol{k}}{k} U_{\mathrm{v}}^{\mathrm{F}}(k) .
$$

This potential is applied to the spinor $u(k)$, eq. (2.27). The Dirac equation (2.26) gives

$$
\boldsymbol{\gamma} \cdot \boldsymbol{k} u(\boldsymbol{k})=\frac{\boldsymbol{k}^{2}}{\boldsymbol{k} \cdot \boldsymbol{k}_{\mathrm{v}}}\left[\gamma^{\mathrm{O}}\left(E_{k}-U_{0}^{\mathrm{HF}}\right)-m_{\mathrm{HF}}(k)\right] u(k) .
$$

Using this result in eq. (2.38) we obtain the following relativistic potential which is fully equivalent to $U^{\mathrm{HF}}(k)$ and which is the sum of only a scalar and the fourth component of a vector:

$$
U^{\mathrm{HF}}(k)=I\left\{U_{\mathrm{s}}^{\mathrm{HF}}(\mathrm{k})-\frac{k m_{\mathrm{HF}}(k)}{\boldsymbol{k} \cdot \boldsymbol{k}_{\mathrm{v}}} U_{\mathrm{v}}^{F}(k)\right\}+\gamma^{0}\left\{U_{0}^{\mathrm{HF}}(k)+\frac{k\left[E_{k}-U_{0}^{\mathrm{HF}}(k)\right]}{\boldsymbol{k} \cdot \boldsymbol{k}_{\mathrm{v}}} U_{\mathrm{v}}^{\mathrm{F}}(k)\right\} .
$$

Conversely, some treatments lead to an expression for the relativistic singleparticle field which is the sum of four terms with well-defined Lorentz transformation properties. This would for instance result from the application of Miller's transformation ${ }^{20}$ ), as we now show. One can use the identity

$$
u^{(\mathrm{s})}(k) u^{(\mathrm{s})}(\boldsymbol{k})^{\dagger} u^{(\mathrm{s})}(\boldsymbol{k})=\delta_{\mathrm{ss}} u^{(\mathrm{s})}(\boldsymbol{k})
$$

to define a potential $\tilde{U}^{\mathrm{HF}}(\boldsymbol{k})$ which is fully equivalent to $U^{\mathrm{HF}}(\boldsymbol{k})$, in the sense that

$$
\begin{gathered}
U^{\mathrm{HF}}(\boldsymbol{k}) u^{(\mathrm{S})}(\boldsymbol{k})=\tilde{U}^{\mathrm{HF}}(\boldsymbol{k}) u^{(\mathrm{S})}(\boldsymbol{k}), \\
\tilde{U}^{\mathrm{HF}}(\boldsymbol{k})=U^{\mathrm{HF}}(\boldsymbol{k}) \sum_{\mathrm{s}} u^{(\mathrm{s})}(\boldsymbol{k}) u^{(\mathrm{s})}(\boldsymbol{k})^{+} .
\end{gathered}
$$

One can then use the following relation ${ }^{1}$ )

$$
u^{(\mathrm{s})}(\boldsymbol{k}) u^{(\mathrm{s})}(\boldsymbol{k})^{\dagger}=\frac{1}{4} \sum_{A=1}^{16}\left[u^{(\mathrm{s})}(\boldsymbol{k})^{\dagger} \gamma_{\mathrm{A}} u^{(\mathrm{s})}(\boldsymbol{k})\right] \gamma^{\mathrm{A}},
$$

where the matrices $\gamma^{A}$ are the standard linearly independent products of Dirac matrices, namely $\gamma^{A}=I, \gamma^{\mu}, \gamma^{5}, \gamma^{5} \gamma^{\mu}, \sigma^{\mu \nu}$. One finds that $\tilde{U}^{\mathrm{HF}}$ does not have the form (2.38). One has

$$
\sum_{\mathrm{s}} u^{(\mathrm{s})}(\boldsymbol{k}) u^{(\mathrm{s})}(\boldsymbol{k})^{\dagger}=\frac{1}{2}\left\{I+\frac{m_{\mathrm{HF}}(k)}{\left[k_{\mathrm{v}}^{2}+m_{\mathrm{HF}}^{2}\right]^{1 / 2}} \gamma^{0}+\frac{\boldsymbol{\alpha} \cdot \boldsymbol{k}_{\mathrm{v}}}{\left[k_{\mathrm{v}}^{2}+m_{\mathrm{HF}}^{2}\right]^{1 / 2}}\right\} .
$$

Eqs. (2.44) and (2.45) give $\tilde{U}^{\mathrm{HF}}$ in the form

$$
\tilde{U}^{\mathrm{HF}}(\boldsymbol{k})=\tilde{U}_{\mathrm{s}}^{\mathrm{HF}}(k)+\gamma^{0} \tilde{U}_{0}^{\mathrm{HF}}(k)+\boldsymbol{\gamma} \cdot \frac{\boldsymbol{k}}{k} \tilde{U}_{\mathrm{v}}^{\mathrm{HF}}(k)+\gamma_{0} \boldsymbol{\gamma} \cdot \frac{\boldsymbol{k}}{k} \tilde{U}_{\mathrm{t}}^{\mathrm{HF}}(k) .
$$

By using a procedure analogous to the one which leads from eq. (2.38) to eq. (2.40), one can show that in the case of symmetric nuclear matter it is possible to find a potential which is fully equivalent to the most general form (2.46) and which has either one of the simple forms $I a(k)+\gamma^{0} b(k)+\gamma_{0} \gamma \cdot k c(k)$, or $I d(k)+\gamma \cdot k e(k)+$ $\gamma_{0} \boldsymbol{\gamma} \cdot \boldsymbol{k} f(k)$, or $\boldsymbol{\gamma}^{0} g(k)+\boldsymbol{\gamma} \cdot \boldsymbol{k} h(k)+\gamma_{0} \boldsymbol{\gamma} \cdot \boldsymbol{k} j(k)$.

The existence of various equivalent values of the relativistic Hartree-Fock field is not surprising since there exists an infinite number of $4 \times 4$ matrices $U$ which are solutions of the equation

$$
U u^{(\mathrm{S})}(\boldsymbol{k})=U^{\mathrm{HF}} u^{(\mathrm{S})}(\boldsymbol{k})=\psi^{(\mathrm{S})}(\boldsymbol{k}),
$$

where $u^{(\mathbf{S})}(\boldsymbol{k})$ and $\psi^{(\mathrm{S})}(\boldsymbol{k})$ are given spinors. Indeed, eq. (2.47) represents a set of only four equations for the 16 unknown components of $U$. We emphasize that all these relativistic (matrix) potentials are fully equivalent in the sense that they yield the same values for all physical observables. The flexibility of the choice of the singleparticle potential may be of practical interest. Indeed, it might be possible that one form of the potential exists in which the Lorentz components are all small. This would greatly facilitate the derivation of a non-relativistic limit. In the following, we shall for definiteness adopt the representation specified by eqs. (2.30)-(2.38).

\subsection{AVERAGE ENERGY PER NUCLEON}

In the Hartree-Fock mean field approximation, the potential energy of a nucleon with momentum $\boldsymbol{k}$ reads

$$
\mathcal{U}(k)=u(\boldsymbol{k})^{\dagger} U^{\mathrm{HF}} u(\boldsymbol{k})=U_{0}^{\mathrm{HF}}(k)+\frac{U_{\mathrm{s}}^{\mathrm{HF}}(k) m_{\mathrm{HF}}+U_{\mathrm{v}}^{\mathrm{F}}(k) \boldsymbol{k}_{\mathrm{v}} \cdot \boldsymbol{k} / k}{\left[\boldsymbol{k}_{\mathrm{v}}^{2}+m_{\mathrm{HF}}^{2}\right]^{1 / 2}} .
$$

The kinetic + rest mass energy of nucleon $\boldsymbol{k}$ is given by

$$
\mathscr{K}(k)=u(\boldsymbol{k})^{\dagger}\left(\boldsymbol{\alpha} \cdot \boldsymbol{k}+\gamma^{0} m\right) u(\boldsymbol{k})=\left(m m_{\mathrm{HF}}+\boldsymbol{k}_{\mathrm{v}} \cdot \boldsymbol{k}\right)\left(\boldsymbol{k}_{\mathrm{v}}^{2}+m_{\mathrm{HF}}^{2}\right)^{-1 / 2} .
$$

The average energy per nucleon is obtained by substituting these expressions in eq. (2.23). 


\section{Schrödinger-equivalent potential}

\subsection{INTRODUCTION}

The Dirac equation (2.6a) can be solved to find the single-particle bound state energies $E_{k}$ and wave functions $\phi_{k}(r)$, from which the ground state properties of nuclei can be investigated ${ }^{1,9,17}$ ). In the case of positive energies, one can also solve the four-component Dirac equation (2.6a) in order to obtain the scattering spinor $\phi_{k}(\boldsymbol{r})$ at energy $E_{k}$ from which elastic scattering phase shifts can be obtained and compared with experimental values ${ }^{12}$ ). However, the comparison between a theoretical approach and nuclear reaction data usually proceeds via an intermediate quantity, namely the average nucleon-nucleus potential. The latter is determined empirically from the analysis of the data with a non-relativistic two-component Schrödinger equation. The purpose of the present section is to construct a nonrelativistic $2 \times 2$ potential, which can thus be used in conjunction with a Schrödinger equation, and which yields exactly the same bound state energies and elastic scattering phase shifts as the relativistic $4 \times 4$ single-particle potential $U^{\mathrm{HF}}$. This "Schrödinger-equivalent potential" can be compared with the real part of the empirical optical-model potential at positive energy, or with the empirical shellmodel potential at negative energy. It has been constructed in refs. ${ }^{9,14,17}$ ) in the case of the Hartree approximation. We shall see that the non-locality which is characteristic of the Hartree-Fock potential introduces some new features.

\subsection{FINITE NUCLEI}

It has been shown by Miller ${ }^{20}$ ) that one can replace the non-local potential $U^{\mathrm{HF}}\left(\boldsymbol{r}, \boldsymbol{r}^{\prime}\right)$ by a local but state-dependent potential $U^{(k)}(r)$. For simplicity we drop the upper index $(k)$ which refers to the state dependence. In the case of doubly closed shell nuclei, the Dirac-Hartree-Fock equation then reads ${ }^{1}$ )

$$
\left\{\boldsymbol{\alpha} \cdot \boldsymbol{p}+\gamma^{0}\left[m+U_{\mathrm{s}}(r)+\gamma^{0} U_{0}(r)+\boldsymbol{\gamma} \cdot \boldsymbol{U}_{\mathrm{v}}(r)\right]\right\} \phi=E \phi,
$$

with $\boldsymbol{U}_{\mathrm{v}}(r)=U_{\mathrm{v}}(r) \boldsymbol{r} / r$. Let us write

$$
\phi=\left(\begin{array}{c}
\tilde{\phi}_{>} \\
\tilde{\phi}_{<}
\end{array}\right)
$$

where $\tilde{\phi}_{>}$and $\tilde{\phi}_{<}$denote the large and the small components of $\phi$, respectively. The Dirac equation (3.1) yields the following two coupled equations

$$
\begin{aligned}
& \boldsymbol{\sigma} \cdot\left[\boldsymbol{p}+\boldsymbol{U}_{\mathrm{v}}(r)\right] \tilde{\phi}_{<}+\left[m+U_{\mathrm{s}}(r)+U_{0}(r)-E\right] \tilde{\phi}_{>}=0, \\
& \boldsymbol{\sigma} \cdot\left[\boldsymbol{p}+\boldsymbol{U}_{\mathrm{v}}(r)\right] \tilde{\phi}_{>}+\left[U_{0}(r)-U_{\mathrm{s}}(r)-E-m\right] \tilde{\phi}_{<}=0,
\end{aligned}
$$

One can eliminate the small components $\tilde{\phi}_{<}$by applying the operator $\boldsymbol{\sigma} \cdot\left[\boldsymbol{p}+\boldsymbol{U}_{\mathrm{v}}(r)\right]$ from the left to eq. (3.3b). After a few manipulations one obtains

$$
\left\{\left[\boldsymbol{p}+\boldsymbol{U}_{\mathrm{v}}(r)\right]^{2}+2 m\left[U_{\mathrm{e}}(r ; \varepsilon)+U_{\text {s.o. }}(r ; \varepsilon) \frac{\boldsymbol{\sigma} \cdot \boldsymbol{L}}{r}+\tilde{C}(r ; \varepsilon)\right]\right\} \tilde{\phi}_{>}=k_{\infty}^{2} \tilde{\phi}_{>},
$$

with $\varepsilon=E-m$ while $k_{\infty}^{2}=2 m \varepsilon+\varepsilon^{2}$ is the momentum of the incident nucleon and

$$
\begin{aligned}
U_{\mathrm{e}}(r ; \varepsilon) & =U_{\mathrm{s}}(r)+U_{0}(r)+\frac{U_{\mathrm{s}}^{2}(r)}{2 m}-\frac{U_{0}^{2}(r)}{2 m}+U_{0}(r) \frac{\varepsilon}{m}, \\
U_{\mathrm{s} . \mathrm{o} .}(r ; \varepsilon) & =-(2 m)^{-1} \frac{\partial}{\partial r}\left[\ln \left(-U_{0}(r)+U_{\mathrm{s}}(r)+E+m\right)\right], \\
\tilde{C}(r ; \varepsilon) & =-i r^{-1} U_{\mathrm{s} . \mathrm{o}}(r ; \varepsilon)\left[\boldsymbol{r} \cdot \boldsymbol{p}+r U_{\mathrm{v}}(r)\right] .
\end{aligned}
$$

Eq. (3.4) can be written in the form

$$
\left(\frac{p^{2}}{2 m}+\tilde{U}_{\mathrm{e}}+U_{\text {s.o. }} \frac{\boldsymbol{\sigma} \cdot \boldsymbol{L}}{r}\right) \tilde{\phi}_{>}=\frac{k_{\infty}^{2}}{2 m} \tilde{\phi}_{>}
$$

where

$$
\tilde{U}_{\mathrm{e}}=U_{\mathrm{e}}+\tilde{C}+(2 m)^{-1}\left[U_{\mathrm{v}}^{2}+\left\{\boldsymbol{p}, \boldsymbol{U}_{\mathrm{v}}\right\}\right] .
$$

Eq. (3.8) formally resembles the Schrödinger equation. However, we point out that $\tilde{U}_{\mathrm{e}}$ involves the operator $\boldsymbol{p}=-i \boldsymbol{\nabla}$. Hence, $\tilde{U}_{\mathrm{e}}$ is not a local operator and is therefore not comparable with the phenomenological local nucleon-nucleus potential.

In order to obtain a Schrödinger-type equation with a local potential, let us introduce the function

$$
\phi_{>}(\boldsymbol{r})=\left\{\exp i \int_{0}^{r} U_{\mathrm{v}}(t) \mathrm{d} t\right\} \tilde{\phi}_{>}(\boldsymbol{r}) .
$$

One can easily check that $\phi_{>}$fulfills the equation

$$
\left(\frac{\boldsymbol{p}^{2}}{2 m}+U_{\mathbf{e}}(r ; \varepsilon)+U_{\text {s.o. }}(r ; \varepsilon) \frac{\boldsymbol{\sigma} \cdot \boldsymbol{L}}{r}+C(r ; \varepsilon)\right) \phi_{>}=k_{\infty}^{2} \phi_{>},
$$

where

$$
C(r ; \varepsilon)=-i r^{-1} U_{\text {s.o. }}(r ; \varepsilon) r \cdot p .
$$

We note that the derivative (and thus non-local) term $C(r ; \varepsilon)$ is present even in the Hartree approximation. Another transformation than (3.10) exists which yields a local potential. We do not give it here since $C(r ; \varepsilon)$ is in practice negligible. We henceforth omit it. Eq. (3.11) is now a Schrödinger-type equation which involves the local potential $U_{\mathrm{e}}(r ; \varepsilon)$. It is thus the latter quantity that we should compare with the real part of the phenomenological nucleon-nucleus potential. Indeed, we emphasize that the wave functions $\tilde{\phi}_{>}(r ; \varepsilon)$ and $\phi_{>}(r ; \varepsilon)$ have the same asymptotic behaviour for large $r$, and therefore the same elastic scattering phase shifts. Moreover, the 
negative (bound) eigenvalues of eqs. (3.4) and (3.11) are equal. Finally we note that, in contradistinction with $\tilde{U}_{\mathrm{e}}$, the local potential $U_{\mathrm{e}}(r ; \varepsilon)$ [eq. (3.5)] does not involve the quantity $U_{\mathrm{v}}(r)$. This is in keeping with the finding of Miller ${ }^{18}$ ) that $U_{\mathrm{v}}(r)$ does not influence the single-particle energies.

\subsection{NUCLEAR MATTER}

In nuclear matter, the Dirac-Hartree-Fock equation (3.1) reduces to eq. (2.26), that we write in the more explicit form

$$
\left\{\boldsymbol{\alpha} \cdot \boldsymbol{k}+\gamma^{0}\left[m+U_{\mathrm{s}}^{\mathrm{HF}}(k)+\gamma^{0} U_{0}^{\mathrm{HF}}(k)+\boldsymbol{\gamma} \cdot \frac{\boldsymbol{k}}{k} U_{\mathrm{v}}^{\mathrm{F}}(k)\right]\right\} u(\boldsymbol{k})=E_{k} u(k) .
$$

In analogy with the procedure used in subsect. 3.2, let us search for an equation for the large components of the spinor

$$
u(k)=\left(\begin{array}{l}
u_{>} \\
u_{<}
\end{array}\right)
$$

One obtains after a few manipulations

$$
\left[\left(m+U_{\mathrm{s}}^{\mathrm{HF}}\right)^{2}-\left(E_{k}-U_{0}^{\mathrm{HF}}\right)^{2}+k_{\mathrm{v}}^{2}\right] u_{>}=0,
$$

i.e. the energy-momentum relation (2.32). This can be written in the Schrödingertype form

$$
\frac{k^{2}}{2 m}+\tilde{U}_{\mathrm{e}}\left(\varepsilon_{k}\right)=\frac{k_{\infty}^{2}}{2 m}
$$

where $k_{\infty}$ is the momentum of a free nucleon with energy $E_{k}=\varepsilon_{k}+m$, while

$$
\begin{gathered}
\tilde{U}_{\mathrm{e}}\left(\varepsilon_{k}\right)=U_{\mathrm{e}}\left(\varepsilon_{k}\right)+\frac{k}{m} U_{\mathrm{v}}^{\mathrm{F}}+\frac{1}{2 m}\left(U_{\mathrm{v}}^{\mathrm{F}}\right)^{2}, \\
U_{\mathrm{e}}\left(\varepsilon_{k}\right)=U_{\mathrm{s}}^{\mathrm{HF}}(k)+U_{0}^{\mathrm{HF}}(k)+(2 m)^{-1}\left\{\left[U_{\mathrm{s}}^{\mathrm{HF}}(k)\right]^{2}-\left[U_{0}^{\mathrm{HF}}(k)\right]^{2}\right\}+\frac{\varepsilon_{k}}{m} U_{0}^{\mathrm{HF}}(k) .
\end{gathered}
$$

This notation is consistent with the one used in eqs. (3.5)-(3.9) in the case of finite nuclei. In particular, we note that $\tilde{U}_{\mathrm{e}}$ as defined by eq. (3.9) reduces to $\tilde{U}_{\mathrm{e}}\left(\varepsilon_{k}\right)$ in the case of a uniform medium. Our discussion in subsect. 3.2 indicates that it would be inappropriate to identify the quantity $\tilde{U}_{\mathrm{e}}\left(\varepsilon_{k}\right)$ with the depth of the potential felt by a nucleon with energy $E_{k}=\varepsilon_{k}+m$. Indeed, we argued in connection with eq. (3.9) that $\tilde{U}_{\mathrm{e}}$ is not a local operator. This is reflected in eq. (3.17) by the appearance in front of $U_{\mathrm{v}}^{\mathrm{F}}$ of the factor $k / m$ which corresponds to a derivative [of exp (ikr)] term in the potential field. Our derivation of the Schrödinger-type equation (3.11) demonstrates that the quantity. which should be identified with the depth of the local nonrelativistic potential felt by a nucleon with energy $E_{k}$ is the Schrödinger-equivalent potential $U_{\mathrm{e}}\left(\varepsilon_{k}\right)$ defined by eq. (3.18). We note that the quantity $U_{\mathrm{e}}\left(\varepsilon_{k}\right)$ does not explicitly involve the component $U_{\mathrm{v}}^{\mathrm{F}}(k)$ of the relativistic potential, although the latter slightly influences $U_{\mathrm{e}}\left(\varepsilon_{k}\right)$ via the energy-momentum relation (3.15) which connects $k$ and $\varepsilon_{k}$

\subsection{LOCAL DENSITY APPROXIMATION}

We now briefly indicate one simple way of constructing a local nucleon-nucleus potential from the nuclear matter results. The quantity $U_{\mathrm{e}}\left(\varepsilon_{k}\right)$ defined in eq. (3.18) depends on the nucleon energy $E_{k}=\varepsilon_{k}+m$ and on the Fermi momentum $k_{\mathrm{F}}$. Let us explicitly indicate the latter dependence by writing

$$
U_{\mathrm{e}}(\varepsilon)=U_{\mathrm{e}}\left(k_{\mathrm{F}} ; \varepsilon\right),
$$

where for simplicity we dropped the index $k$ on $\varepsilon_{k}$. The simplest local density approximation consists in relating $k_{\mathrm{F}}$ to the radial distance $r$ from the nuclear centre by the equation

$$
\rho(r)=\frac{2}{3 \pi^{2}} k_{\mathrm{F}}^{3}(r)
$$

where $\rho(r)$ is the empirical matter distribution in the target nucleus. This yields the following approximation for the local Schrödinger-equivalent potential in a finite nucleus

$$
U_{\mathrm{e}}(r ; \varepsilon)=U_{\mathrm{e}}\left(k_{\mathrm{F}}(r) ; \varepsilon\right)
$$

Other local density approximations could be used. For instance, one could fold the contribution $U_{\mathrm{s}}^{\sigma}$ of the $\sigma$-meson to $U_{\mathrm{s}}^{\mathrm{HF}}$ according to the recipe

$$
U_{\mathrm{s}}^{\sigma}(r)=C_{\mathrm{s}}^{\sigma} \int \mathrm{d}^{3} \boldsymbol{r}^{\prime} U_{\mathrm{s}}^{\sigma}\left(k_{\mathrm{F}}\left(\boldsymbol{r}^{\prime}\right)\right)\left|\boldsymbol{r}-\boldsymbol{r}^{\prime}\right|^{-1}\left\{\exp \left(-\left|\boldsymbol{r}-\boldsymbol{r}^{\prime}\right| m_{\sigma}\right)-\exp \left(-\left|\boldsymbol{r}-\boldsymbol{r}^{\prime}\right| \Lambda_{\sigma}\right)\right\},
$$

where $C_{\mathrm{s}}^{\sigma}$ is a normalization constant, and correspondingly with the other contributions ${ }^{21}$ ). This folding recipe is an improvement over the simple local-density approximation (3.21) because it includes the effect of the range of the effective nucleon-nucleon interaction ${ }^{22}$ ).

\section{Numerical results}

\subsection{INPUT PARAMETERS}

In ref. ${ }^{14}$ ) we presented some numerical results obtained in the framework of the relativistic Hartree mean field approximation. In order to illustrate the modifications 
introduced by the Fock contribution, we shall below usually adopt the same input parameters as in ref. ${ }^{14}$ ), namely

$$
\begin{array}{ccc}
m_{\sigma}=550 \mathrm{MeV}, & g_{\sigma}^{2} / 4 \pi=6.57, & \Lambda_{\sigma}=1530 \mathrm{MeV}, \\
m_{\omega}=782.8 \mathrm{MeV}, & g_{\omega}^{2} / 4 \pi=9.25, & \Lambda_{\omega}=1530 \mathrm{MeV} .
\end{array}
$$

This parameter set is identical to the one used by Brockmann ${ }^{9}$ ) in his investigation of ${ }^{16} \mathrm{O}$ and of ${ }^{40} \mathrm{Ca}$. In subsects. 4.3-4.5, we shall use another set of parameters, chosen in such a way that the empirical saturation point of nuclear matter coincides with that calculated from the relativistic Hartree-Fock mean field approximation.

\subsection{RELATIVISTIC SINGLE-PARTICLE POTENTIAL}

The relativistic Hartree-Fock mean field $U^{\mathrm{HF}}$ is a $4 \times 4$ matrix which can be written in the form (2.38). The direct part $U^{\mathrm{H}}$ has been calculated in ref. ${ }^{14}$ ) in the framework of the Hartree mean field approximation. Its scalar component $U_{\mathrm{s}}^{\mathrm{H}}$ is now slightly modified because it involves the Hartree-Fock effective mass $m_{\mathrm{HF}}$ [eq. (2.29)] instead of the Hartree effective mass $m_{\mathrm{H}}$. At $k_{\mathrm{F}}=1.35 \mathrm{fm}^{-1}$ for instance, $U_{\mathrm{s}}^{\mathrm{H}}$ is found equal to $-325.6 \mathrm{MeV}$ in the case of the relativistic Hartree-Fock mean field approximation, while in ref. ${ }^{14}$ ) we had found $U_{\mathrm{s}}^{\mathrm{H}}=-329 \mathrm{MeV}$ in the framework of the relativistic Hartree mean field approximation. Note that the first-order relativistic Hartree approximation, which consists in setting $m_{\mathrm{H}}=m$ in eq. (2.17), would yield $U_{\mathrm{s}}^{\mathrm{H}}=-338.8 \mathrm{MeV}$. This illustrates the role of the self-consistent requirements.

We now focus on the Fock components. In fig. 1, we show the value of the scalar component $U_{\mathrm{s}}^{\mathrm{F}}(k)$. It is the sum of two contributions due to $\sigma$ - and to $\omega$-exchange, respectively. Note that the vector meson $\omega$ yields a contribution to the scalar component of the Fock potential, while in the case of the relativistic Hartree mean

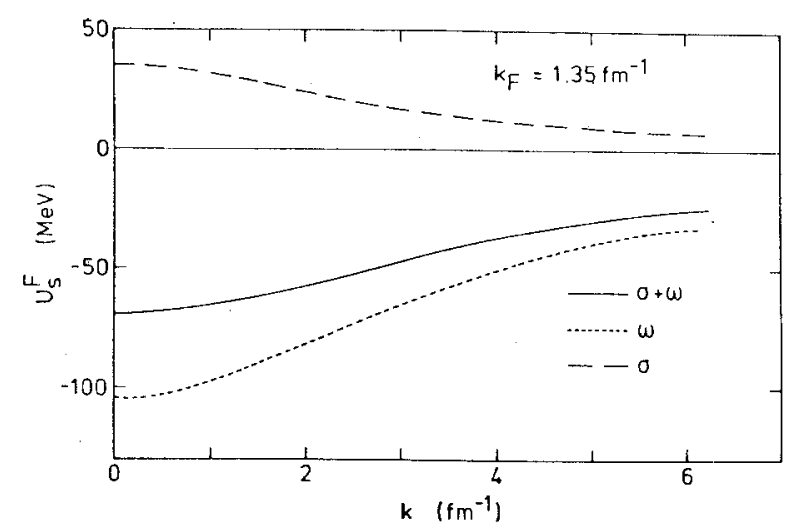

Fig. 1. The full curve represents the dependence upon $k$ of the scalar component $U_{\mathrm{s}}^{F}(k)$ of the Fock contribution, for $k_{\mathrm{F}}=1.35 \mathrm{fm}^{-1}$ and for the input parameters (4.1). The long dashes and the short dashes show the contributions to $U_{\mathrm{s}}^{\mathrm{F}}(k)$ of $\sigma$-exchange and of $\omega$-exchange, respectively. field approximation the vector meson only contributes to the quantity $U_{0}$. The quantity $U_{\mathrm{s}}^{\mathbf{F}}(k)$ tends towards zero as $k \rightarrow \infty$, as expected from a Fock (exchange) contribution.

Fig. 2 shows the value of the Fock contribution to the fourth component $U_{0}^{\mathrm{F}}(k)$ of the vector field. It is the sum of two contributions respectively associated with

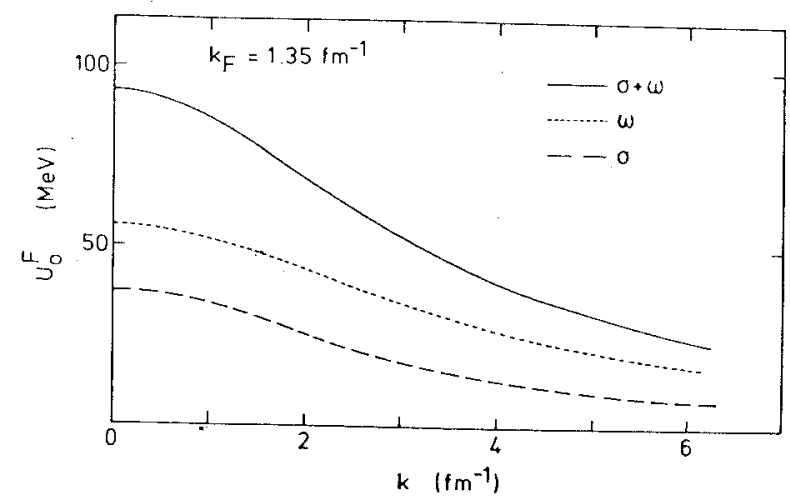

Fig. 2. Same as fig. 1, in the case of the quantity $U_{0}^{F}(k)$ which is the fourth component of the vector part of the relativistic Fock potential.

$\omega$-exchange and with $\sigma$-exchange. The quantity $U_{\mathrm{v}}^{\mathrm{F}}(k)$ shown in fig. 3 corresponds to the spatial components of the vector part of the relativistic mean field. These components vanish in the case of the Hartree mean field approximation which has been considered in ref. $\left.{ }^{14}\right)$. The value of $U_{\mathrm{v}}^{\mathrm{F}}(k)$ is much smaller than those of $U_{\mathrm{s}}^{\mathrm{F}}(k)$ and of $U_{0}^{\mathrm{F}}(k)$. We moreover recall [see eq. (3.11)] that in the case of a finite nucleus $U_{\mathrm{v}}^{\mathrm{F}}$ does not affect at all the value of the single-particle bound state energies nor of the elastic scattering phase shifts, no matter how large $U_{\mathrm{v}}^{\mathrm{F}}$ is. We note, however, that $U_{\mathrm{v}}^{\mathrm{F}}$ influences the value of the single-particle wave function at finite distance.

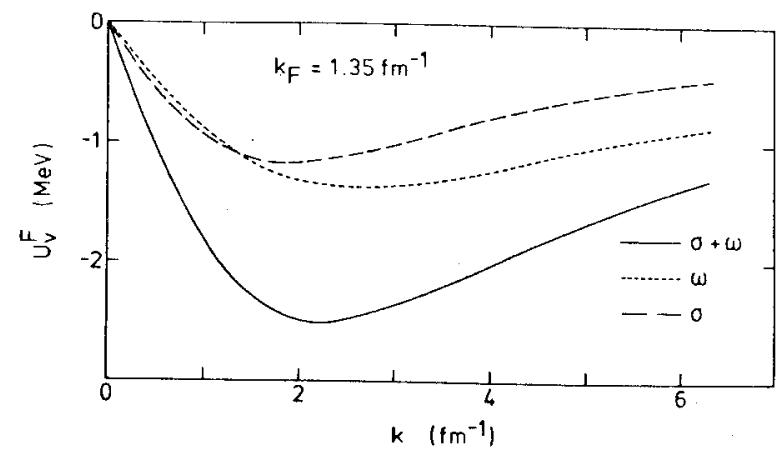

Fig. 3. Same as fig. 1, in the case of the quantity $U_{\mathrm{v}}^{\mathrm{F}}(k)$ which corresponds to the spatial components of the vector part of the relativistic Fock potential. 


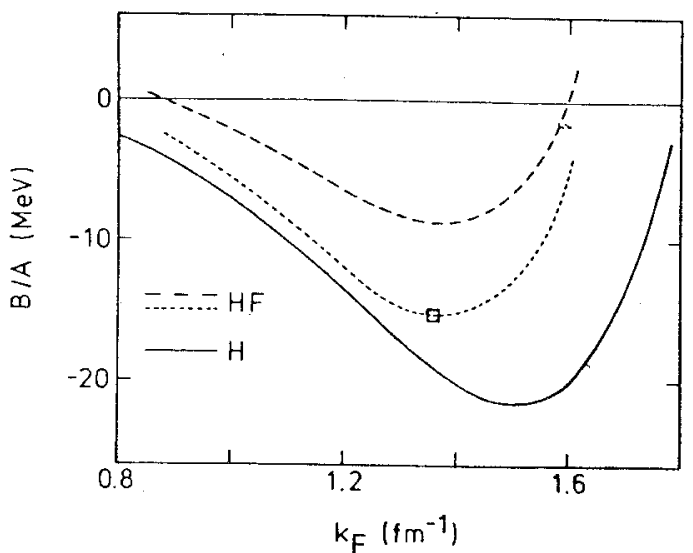

Fig. 4. Average binding energy per nucleon as calculated from the relativistic Hartree mean field approximation (full curve) and from the relativistic Hartree-Fock mean field approximation (long dashes) for the input parameters (4.1). The short dashes correspond to the Hartree-Fock resul input parameters (4.2). The empirical saturation point is represented by an open square.

\subsection{BINDING ENERGY}

In the case of the relativistic Hartree mean field approximation, the average binding energy per nucleon has been calculated in ref. ${ }^{14}$ ) with the input parameters (4.1); its dependence upon the Fermi momentum is represented by the full curve in fig. 4. There, the long dashes show the average binding energy per nucleon as calculated from the relativistic Hartree-Fock mean field approximation, again for the input parameters (4.1). We note that the Fock contribution yields a significant contribution to $B / A$.

The input parameters are fitted to some observables and depend upon the approximation (e.g. Hartree or Hartree-Fock) which is used. They moreover contain renormalization corrections. Hence, they should not be directly identified with the parameters encountered in one-boson exchanged potentials. They should rather be considered as effective coupling constants and masses. It is possible to choose the input parameters in such a way that the empirical saturation point $(B / A=$ $\left.-15.6 \mathrm{MeV}, k_{\mathrm{F}}=1.36 \mathrm{fm}^{-1}\right)$ is exactly reproduced by the relativistic Hartree-Fock mean field approximation. Note that in the latter $B / A$ depends upon all six input parameters, while it only depends upon the two quantities $g_{\sigma} / m_{\sigma}$ and $g_{\omega} / m_{\omega}$ in the Hartree mean field approximation ${ }^{3}$ ). Here, we choose to adjust the coupling constants $g_{\sigma}$ and $g_{\omega}$. The short dashes in fig. 4 correspond to the Hartree-Fock mean field approximation with the following values for the input parameters

$$
\begin{array}{cll}
m_{\sigma}=550 \mathrm{MeV}, & g_{\sigma}^{2} / 4 \pi=7.47, & \Lambda_{\sigma}=1530 \mathrm{MeV}, \\
m_{\omega}=782.8 \mathrm{MeV}, & g_{\omega}^{2} / 4 \pi=10.15, & \Lambda_{\omega}=1530 \mathrm{MeV} .
\end{array}
$$

We note that the parameter sets (4.1) and (4.2) are not very different. It is also striking that these values are close to those which appear in one-boson exchange potentials for free nucleon-nucleon scattering ${ }^{9}$ ). These effective meson parameters will of course change if one considers the exchange of other mesons or if one considers some effects of the nucleon-nucleon correlations ${ }^{15,17}$ ). However, their order of magnitude is unlikely to be affected. Hence, we expect that relativistic effects, such as those considered in the present paper, will remain quite sizeable.

In the relativistic Hartree-Fock approximation, each nucleon with momentum $k<k_{\mathrm{F}}$ can be ascribed a kinetic energy $\mathscr{K}(k)$ and a potential energy $\mathscr{U}(k)$ [eqs. $(2.49),(2.48)]$. The single-particle potential energy $\mathscr{U}(k)$ should not be confused with the Hartree-Fock approximation to the self-energy as defined in many-body theory ${ }^{4,6,23}$ ), i.e. with the $4 \times 4$ matrix $U^{\mathrm{HF}}$, or with the Schrödinger-equivalent potential $U_{\mathrm{e}}$. The nucleon potential energy $U(k)$ as calculated from eq. $(2.48)$ is represented by the full curve in fig. 5 . There, the importance of the self-consistent requirements characteristic of a mean field approximation is illustrated by the difference between the full curve and the dashed curve. The latter is obtained by dropping all the self-consistent requirements, i.e. by performing a lowest-order calculation in the coupling constants.

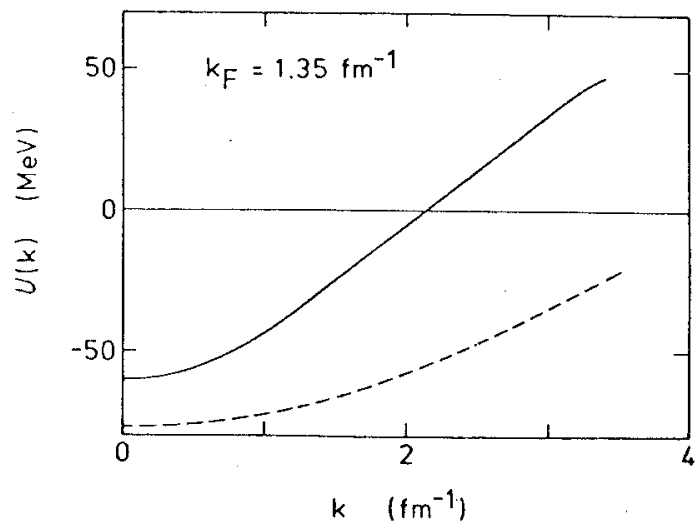

Fig. 5. The full curve shows the potential energy of a nucleon with momentum $k$ as calculated from the input parameters (4.1) in the framework of the relativistic Hartree-Fock mean field approximation. The long dashes are obtained by dropping all the self-consistent requirements.

\subsection{SCHRÖDINGER-EQUIVALENT POTENTIAI}

We showed in subsect. 3.3 that $U_{\mathrm{e}}(\varepsilon)$ can be identified with the depth of the standard (non-relativistic) average potential felt by a nucleon with energy $\varepsilon$ The expression (3.18) of $U_{\mathrm{e}}(\varepsilon)$ is formally identical to the one encountered in the framework of the relativistic Hartree approximation ${ }^{14}$ ). In particular, it does not explicitly depend on the spatial components of the vector part of the relativistic potential. Fig. 6 gives the value of $U_{\mathrm{e}}(\varepsilon)$ as obtained from the parameter set (4.1) in the framework of the relativistic Hartree-Fock mean field approximation. A 


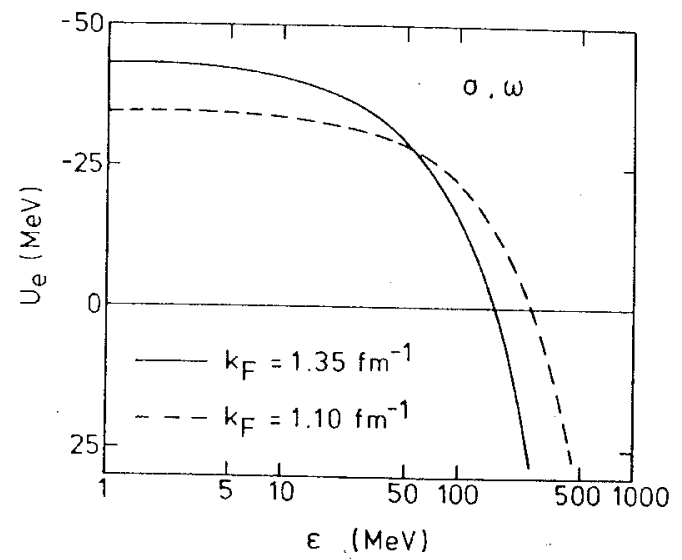

Fig. 6. Dependence upon $\varepsilon$ of the Schrödinger-equivalent potential $U_{\mathrm{c}}(\varepsilon)$ as calculated from the relativistic Hartree-Fock approximation (3.18) with the input parameters (4.1), for the Fermi moment $k_{\mathrm{F}}=1.35 \mathrm{fm}^{-1}$ (full curve) and $k_{\mathrm{F}}=1.10 \mathrm{fm}^{-1}$ (dashed curve), respectively.

comparison with fig. 2 of ref. ${ }^{14}$ ) shows that the present results are less attractive than those derived from the relativistic Hartree mean field approximation with the same input parameters (4.1). This is mainly due to the fact that the attractive Fock component $U_{\mathrm{s}}^{\mathrm{F}}$ is smaller in absolute magnitude than the repulsive Fock component $U_{0}^{\mathrm{F}}$. The $\varepsilon$-dependence of $U_{\mathrm{e}}$ is usually characterized by the quantity

$$
\frac{\bar{m}}{m}=1-\frac{\mathrm{d} U_{\mathrm{e}}(\varepsilon)}{\mathrm{d} \varepsilon} .
$$

For $k_{F}=1.35 \mathrm{fm}^{-1}$ and for the input parameters (4.1), one finds $\bar{m} / m=0.73$ for the Hartree-Fock as well as for the Hartree approximation. This may appear somewhat surprising in view of eq. (3.18) and of the fact that $U_{0}^{\mathrm{HF}}$ is sizeably larger than $U_{0}^{\mathrm{H}}$. However, one must keep in mind that in the Hartree-Fock approximation all the

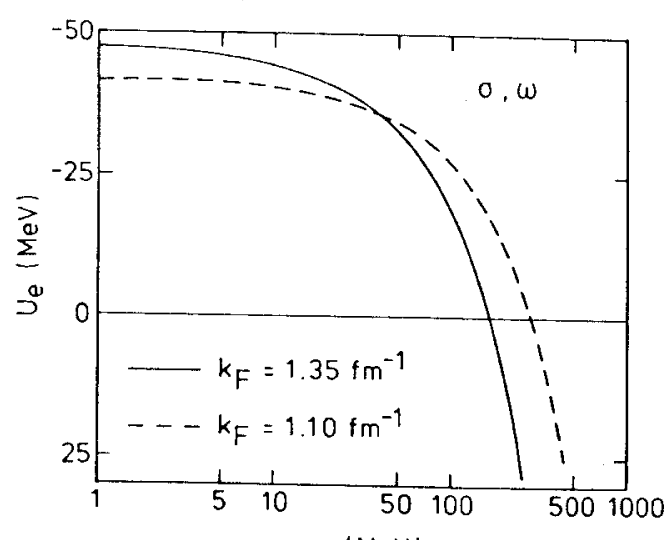

$$
\varepsilon(\mathrm{MeV})
$$

Fig. 7. Same as fig. 6, for the input parameters (4.2). terms on the r.h.s. of eq. (3.18) depend upon $\varepsilon_{k}$. Note that the Schrödingerequivalent potential $U_{\mathrm{e}}$ is quite different from the potential energy $\mathcal{U}$ of a nucleon with the same momentum $k$. This is exhibited by the comparison between figs. 5 and 6.

In the case of the relativistic Hartree-Fock mean field approximation, the parameter set (4.2) may be considered as somewhat more realistic than (4.1) since it reproduces the empirical saturation point of nuclear matter. Fig. 7 shows the corresponding value of $U_{c}(\varepsilon)$; in this case, one finds $\bar{m} / m=0.70$ for $k_{\mathrm{F}}=1.35 \mathrm{fm}^{-1}$. This result is in very good agreement with the energy dependence of the depth of the real part of the empirical optical-model potential.

\subsection{WINE-BOTTLE BOTTOM SHAPE}

Fig. 7 shows that the Schrödinger-equivalent potential changes sign at a lower energy $\left(\varepsilon_{0}=162 \mathrm{MeV}\right)$ for $k_{\mathrm{F}}=1.35 \mathrm{fm}^{-1}$ (which corresponds to the density in the nuclear interior) than for $k_{\mathrm{F}}=1.10 \mathrm{fm}^{-1}\left(\varepsilon_{0}=280 \mathrm{MeV}\right)$ (which corresponds to the density at the nuclear surface). Like in the case of the Hartree mean field approximation $\left.{ }^{14}\right)$, the average nucleon-nucleus potential $U_{\mathrm{e}}(r ; \varepsilon)$ as constructed from the local density approximation (3.21) will therefore display an attractive pocket at the nuclear surface in the intermediate energy range. This "wine-bottle bottom shape" is exhibited by the full curve in fig. 8 , which has been obtained from the parameter set (4.2), and by taking for $\rho(r)$ in eq. (3.20) the nuclear density distribution as parametrized by Negele ${ }^{25}$ ). The origin of the wine-bottle bottom shape is the same as that described in ref. ${ }^{14}$ ) in the case of the Hartree mean field approximation. It lies in the quadratic terms contained inside the curly brackets on the r.h.s. of eq. (3.18), which are typical of a relativistic approach. More specifically, the wine-bottle bottom shape is due to the fact that the content of the curly brackets

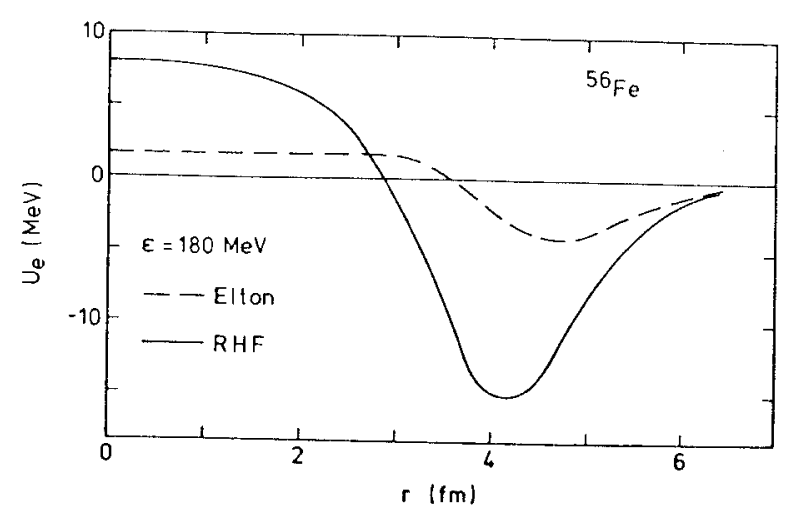

Fig. 8. The full curve represents the Schrödinger-equivalent potential in the case of ${ }^{56} \mathrm{Fe}$ at $\varepsilon=180 \mathrm{MeV}$, as calculated from the local density approximation (3.21) in the framework of the relativistic HartreeFock mean field approximation with the input parameters (4.2); the dashes show the potential well empirically determined by Elton ${ }^{24}$ ) from the analysis of proton scattering data. 
in eq. (3.18) is positive and approximately increases like $\rho^{2}$, while the other terms are proportional to $\rho$. For values of $k_{\mathrm{F}}$ larger than $1.35 \mathrm{fm}^{-1}$, the content of the curly brackets becomes so important that $\left|U_{\mathrm{e}}(\varepsilon=0)\right|$ then decreases with increasing $k_{\mathrm{F}}$. For instance, $U_{\mathrm{e}}(\varepsilon=0)$ is found equal to $-32.8 \mathrm{MeV}$ for $k_{\mathrm{F}}=1.50 \mathrm{fm}^{-1}$ and to $-34.5 \mathrm{MeV}$ for $k_{\mathrm{F}}=1.35 \mathrm{fm}^{-1}$, for the Hartree-Fock mean field approximation with the input parameters (4.1).

The wine-bottle bottom shape which had been found at intermediate energy in the framework of the relativistic Hartree mean field approximation is thus maintained in the relativistic Hartree-Fock mean field approximation. It is even slightly more pronounced in the latter case because the quadratic terms in eq. (3.18) are relatively more important. If the difference between the masses $m_{\sigma}$ and $m_{\omega}$ of the two mesons would be taken into account, for instance by using the folding prescription (3.22) $\left[\right.$ ref. $\left.\left.{ }^{26}\right)\right]$, the attractive surface pocket would become somewhat wider and would be slightly shifted towards the nuclear exterior ${ }^{14}$ ).

\section{Summary and conclusions}

In sect. 2, we extended Walecka's Hartree mean field approximation in nuclear matter ${ }^{3}$ ) in order to include the Fock contribution. This was performed in close analogy with the relativistic Hartree-Fock approximation for finite nuclei studied by Miller and Green ${ }^{1}$ ) and by Brockmann and Weise ${ }^{9,17}$ ). The nature of the selfconsistency requirements characteristic of a mean field approach to nuclear matter theory were stressed and their quantitative importance has been illustrated in sect. 4 .

The structure of the relativistic single-particle potential is qualitatively different in the case of the Hartree-Fock than in the case of the Hartree mean field approximation. The main differences consist in the fact that all the components of the relativistic single-particle potential are non-local, and in the appearance of non-vanishing spatial components for the vector part of the potential; the latter statement must be taken with some caution because in nuclear matter there exist several ways of writing the Hartree-Fock potential as a sum of terms which have well-defined Lorentztransformation properties (subsect. 2.4). These differences have been taken into account in sect. 3 where we constructed a non-relativistic potential which is local and equivalent to the relativistic Hartree-Fock field, equivalent in the sense that it yields exactly the same bound state energies and elastic scattering phase shifts.

Sect. 4 presents some numerical results. We used two sets of input parameters. The first one is identical to that used in refs. ${ }^{9,14}$ ) in connection with the Hartree mean field approximation. The second one is such that the calculated saturation point of nuclear matter coincides with the empirical value. Our numerical results indicate that the inclusion of the Fock contribution does not affect the semi-quantitative success of the relativistic Hartree approximation ${ }^{3,14}$ ) in accounting for the single-particle properties of nuclear matter and of finite nuclei. Some of our conclusions will admittedly have to be adapted when the model will be progressively improved for nstance by considering the effect of the exchange of mesons other than $\sigma$ and $\omega$, or he effect of nucleon-nucleon correlations ${ }^{15,17}$ ). Nevertheless, the main characeristics of the present relativistic models, namely the appearance of strong fields vith definite Lorentz-transformation properties, is likely to subsist. The existence of hese strong fields seems to imply sizeable relativistic effects, which in the present ontext mainly concern the spin-orbit component of the Schrödinger-equivalent otential, and the existence of a wine-bottle bottom shape for the Schrödinger:quivalent potential at intermediate energy. We believe that a gradual understandng of such relativistic effects may best be achieved by the study of simple models like he one studied in the present work.

\section{References}

1) L.D. Miller and A.E.S. Green, Phys. Rev. C5 (1972) 241

2) L.D. Miller, Phys. Rev. C9 (1974) 537

3) J.D. Walecka, Ann. of Phys. 83 (1974) 491

4) M. Bolsterli, Phys. Rev. D11 (1975) 312

5) R.L. Bowers, A.M. Gleeson and R.D. Pedigo, Phys. Rev. D12 (1975) 3043; D12 (1975) 3056

6) S.A. Chin, Ann. of Phys. 108 (1977) 301

7) J. Boguta and J. Rafelski, Phys. Lett. 71B (1977) 22 ;

J. Boguta and A.R. Bodmer, Nucl. Phys. A292 (1977) 413;

J. Boguta, private communication

8) R. Brockmann and W. Weise, Phys. Rev. C16 (1977) 1282

9) R. Brockmann, Phys. Rev. C18 (1978) 1510

10) J.V. Noble, Phys. Rev. C17 (1978) 2151; Nucl. Phys. A329 (1979) 354; Phys. Lett. 89B (1980) 325

11) F.R. Serr and J.D. Walecka, Phys. Lett. 79B (1978) 10; B.D. Serot, Phys. Lett. 86B (1979) 146;

B.D. Serot and J.D. Walecka, Phys. Lett. 87B (1979) 172;

J.D. Walecka, Phys. Lett. 94B (1980) 293;

R. Rosenfelder, Ann. of Phys. 128 (1980) 188

12) L.G. Arnold, B.C. Clark and R.L. Mercer, Phys. Rev. C19 (1979) 917; and references contained therein

13) M. Jaminon, C. Mahaux and P. Rochus, Phys. Rev. Lett. 43 (1979) 1097

14) M. Jaminon, C. Mahaux and P. Rochus, Phys. Rev. C22 (1980) 2027

15) M.R. Anastasio, L.S. Celenza and C.M. Shakin, Phys. Rev. C23 (1981) 569; Phys Rev. Lett. 45 (1980) 2096

16) R. Rosenfelder, Ann. of Phys. 128 (1980) 188

17) R. Brockmann and W. Weise, submitted for publication

18) L.D. Miller, Ann. of Phys. 91 (1975) 40

19) M. Jaminon, C. Mahaux and P. Rochus, Proc. 1980 Enrico Fermi Summer School (in press)

20) L.D. Miller, Phys. Rev. C12 (1975) 710

21) L.G. Arnold and B.C. Clark, Phys. Lett. 84B (1979) 46

22) J.P. Jeukenne, A. Lejeune and C. Mahaux, Phys. Rev. C16 (1977) 80

23) A.L. Fetter and J.D. Walecka, Quantum theory of many-particle systems (McGraw-Hill, NY 1971)

24) L.R.B. Elton, Nucl. Phys. 89 (1966) 69

25) J.W. Negele, Phys. Rev. C1 (1970) 1260

26) R. Humphreys, Nucl. Phys. A182 (1972) 580 